\title{
THE DISTRIBUTION OF GAS AND LIQUID WITHIN THE CUTTLEBONE
}

\author{
By E. J. Denton and J. B. Gilpin-Brown* \\ The Plymouth Laboratory
}

(Plates I and II and Text-figs. I-7)

We have previously given an account of the general properties of the cuttlebone and the way in which the cuttlefish uses it as a variable buoyancy tank (Denton \& Gilpin-Brown, 1961 $a, b$; Denton, Gilpin-Brown \& Howarth, 1961). Here we shall describe how the liquid and gas which the cuttlebone contains are distributed amongst its hundred or so chambers, and attempt to interpret this distribution in terms of the behaviour of the cuttlefish.

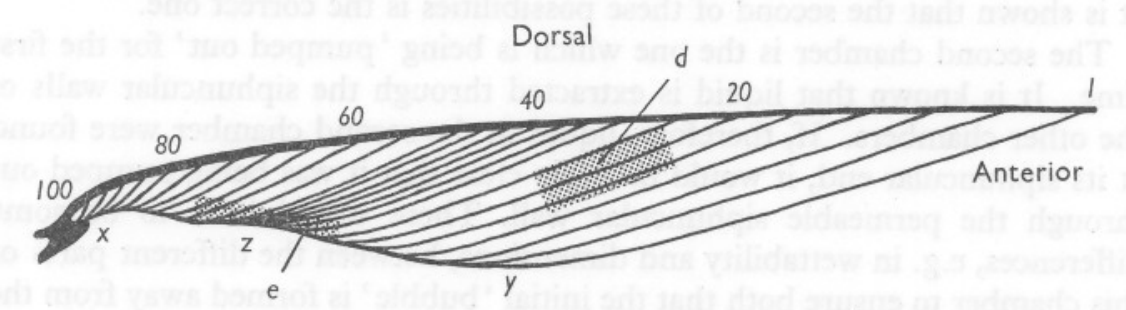

Text-fig. I. Diagram of a cuttlebone (condition in winter) in which only every fourth lamella has been drawn. The true numbers of the chambers are those given along the dorsal surface of the bone. The siphuncular surface is marked $x y$, and it can be seen that the inflexion, $z$, is associated with a number of thin chambers of small volume. The letters $d$ and $e$ refer to the experiment described on p. 368 .

The chambers of the cuttlebone will be numbered from the newest to the oldest chamber (Text-fig. I). Chamber no. I is the incompletely formed chamber lying ventrally and anteriorly; its volume in an adult cuttlefish is between $\mathrm{I}$ and $2 \mathrm{ml}$. The oldest, i.e. the first-formed chamber, is approximately no. 100 and its volume is less than $10^{-3} \mathrm{ml}$. In general the volume of a chamber decreases with increasing number, but on the siphuncular surface of a cuttlebone $(x y)$ a marked inflexion can be seen (this is shown by the letter $z$ in Pl. I and Text-fig. I), and associated with this inflexion there are a number of very thin chambers of small volume. Despite this complication it is quite clear that the newer chambers, having the larger volumes, are those most important in determining the buoyancy of the cuttlefish. The older chambers, although of small volume, have a disproportionate effect on the

\footnotetext{
* Present address: Department of Zoology, The University, Auckland, New Zealand.
} 
posture of the cuttlefish for, since they lie well towards the posterior end of the animal, they will, when gas-filled, tend to make the cuttlefish float tail upwards.

It seems possible that the 'pumping out' of a newly formed chamber might be achieved by a mechanism different from that used to control the amount of liquid in the older ones. The siphuncular walls of the newest few chambers are whiter and more silvery in appearance than the corresponding walls of the older chambers. It was found moreover (Denton \& Gilpin-Brown, I96I $a$ ) that these few are the only chambers which do not lose liquid when the external pressure is reduced. Two simple explanations of this may be suggested:

(i) Liquid is not initially pumped out through the siphuncular wall of a chamber but through the broad ventral wall separating the first and second chambers. The siphuncular walls could, on this hypothesis, be initially impermeable to liquids but become permeable later when six or so more chambers have been added.

(ii) The siphuncular walls of the newest chambers are permeable but the siphuncular ends of these chambers do not contain liquid.

It is shown that the second of these possibilities is the correct one.

The second chamber is the one which is being 'pumped out' for the first time. It is known that liquid is extracted through the siphuncular walls of the other chambers. If, therefore, liquid in the second chamber were found at its siphuncular end, it would be fairly clear that it was being pumped out through the permeable siphuncular wall. There would have to be some differences, e.g. in wettability and dimensions, between the different parts of this chamber to ensure both that the initial 'bubble' is formed away from the siphuncular end and that the column of liquid within the chamber is not completely broken during the pumping out. However, liquid, when present at all in the second chamber, was rarely found at its siphuncular end. The second chamber was indeed the only one in which liquid was not so localized. The following explanations of this may be given:

(i) Liquid is initially extracted through the siphuncular wall but there is no adequate mechanism to ensure that the column of liquid stretching inwards from the siphuncular wall is not broken. The liquid left behind is, however, little by little shaken down to this wall and then extracted. ${ }^{1}$

(ii) Some liquid is extracted through the broad ventral wall of the chamber. Arguing againt this hypothesis is the fact that the living epithelium covering the ventral surface is separated from the wall of the second chamber by the liquidfilled incomplete parts of the first.

(iii) Liquid is first taken from the siphuncular end of the chamber and water then evaporates from the deeper wet parts of the chamber on to the siphuncular surface and is there taken away. The obvious difficulty of this hypothesis is that a progressively stronger salt solution will be left in the deeper parts of the cuttlebone and evaporation will be brought to a stop. We might keep this explanation

${ }^{1}$ Water might be transported by living cells but no such cells could be found within the chambers. 
if: either $(a)$ the liquid in chamber $\mathrm{I}$ were not a salt solution but contained organic substances which were later condensed into the solid parts of the cuttlebone, leaving behind pure water which could be removed by evaporation; or

(b) the salts in the liquid were themselves condensed into the solid structure of the bone leaving pure water behind.

Here it is shown that the water in the first chamber contains sodium and potassium in concentrations close to those of sea water and that these ions are not left behind when the water is initially removed. Since the salts and water leave the newly formed chamber together and, since they are not pushed out by gas pressure, it seems likely that liquid is extracted osmotically. The possibilities listed under (iii) above may therefore be excluded.

It has previously been shown (Denton \& Gilpin-Brown, 1961 $a$ ) that when the cuttlefish changes its density by increasing or decreasing the gas space within its cuttlebone the mass of gas within the cuttlebone remains approximately constant. This has been explained in terms of the osmotic movement of liquid and the slowness of diffusion of gases into and out of the cuttlebone. On this hypothesis we should expect that the pressure of gas would be particularly low in a newly pumped chamber. This is shown to be the case. An estimate of the rate of diffusion of nitrogen (the principal gas in the cuttlebone) into and out of the cuttlebone is made on the basis of an experiment in which the rate of entry of carbon dioxide was studied.

\section{METHODS AND RESULTS}

\section{The permeability of the siphuncular wall}

The following experiment was designed to find out whether or not the siphuncular walls of the newest chambers are permeable to liquid.

Two cuttlebones were taken and the membranes covering their siphuncular surfaces were wiped off. One was placed under sea water for about $2 \mathrm{~h}$. It was then gently dried on a cloth and placed under reduced pressure. Liquid which had soaked into the cuttlebone now came freely from the siphuncular surface, even from the newest chambers (apart from the first incomplete chamber). The second cuttlebone was placed for $30 \mathrm{~min}$ in sea water under a pressure of $+5 \mathrm{~atm}$. When the cuttlebone was brought back to atmospheric pressure, liquid was observed to flow freely from all the newest chambers except the first. These two experiments demonstrate that in all the chambers, except perhaps the newest of all, which is incomplete, the siphuncular surface is freely permeable to water.

Sodium and potassium in different parts of the cuttlebone

An experiment was made to decide between the various mechanisms suggested for the initial pumping out of a newly formed chamber. The following parts of a fresh cuttlebone were scraped off, each into a separate weighing 
bottle: $(a)$ the Ist chamber; $(b)$ the 2 nd chamber; $(c)$ the 3 rd chamber; (d) the anterior regions of some of the older chambers (around the 2oth), see Text-fig. I $;(e)$ the posterior regions close to and including the siphuncular surface, of a number of chambers older than 20 (Text-fig. I). Great care was taken during the scraping to keep the lids on the weighing bottles as much as possible to avoid losses of water by evaporation from the material already collected.

Each of the samples in the weighing bottles was then treated successively as follows: (i) weighed; (ii) extracted with distilled water, and the sodium and potassium content of the extract measured using a flame photometerExtract I ; (iii) dried at $110^{\circ} \mathrm{C}$ to constant weight; (iv) ashed in a silica crucible at $650^{\circ} \mathrm{C}$ to constant weight; (v) the ash extracted with distilled water and after the calcium in the extract had been precipitated with ammonium oxalate the sodium and potassium contents were measuredExtract 2; and (vi) after water extraction, the ash dissolved in strong hydrochloric acid which was then neutralized with ammonium hydroxide, the calcium precipitated with ammonium oxalate, and the total sodium and potassium in the residual solution found-Extract 3 .

TABLE 1 . ANALYSES OF SUCCESSIVE CHAMBERS

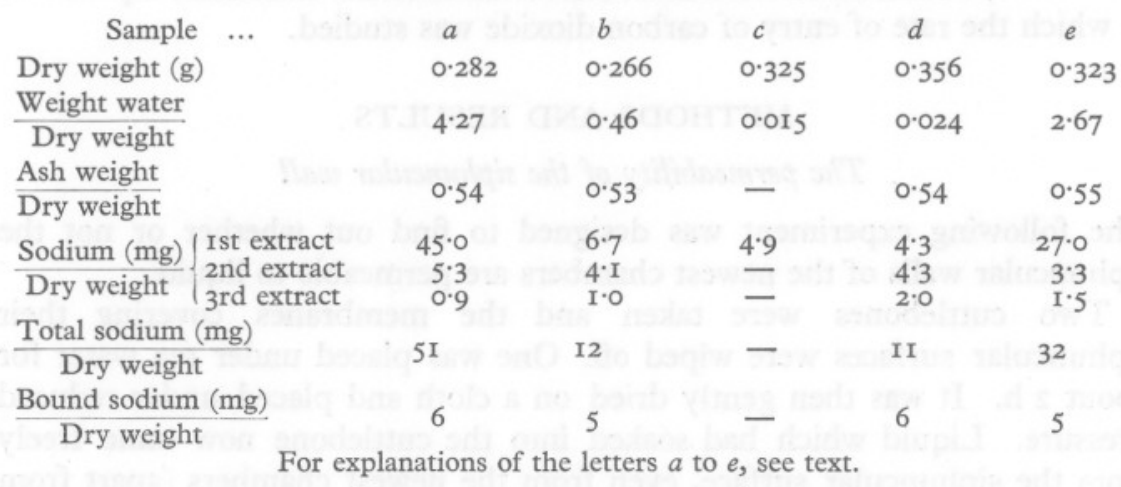

The results of this experiment are summarized in Table $\mathrm{I}$. The weight of potassium in the extracts was always about $5 \%$ of the sodium (in sea water it is about $4 \%$ ). Three conclusions can be drawn from the experiment.

(I) The siphuncular ends of the older chambers (sample $e$ ) can be seen to contain much water, whilst the anterior ends (sample $d$ ) contain very little. This might have been expected, for we have previously found that liquid exchanges in the cuttlebone are made across its siphuncular surface.

(2) All the samples of cuttlebone contained approximately the same proportion of 'bound' sodium and potassium. Here by 'bound' we mean the sodium and potassium which was only extractable after ashing. This amounted to about $10 \%$ of the sodium in the first chamber. Since the solid parts account 
for about $10 \%$ of the total volume of a chamber ${ }^{1}$ the amount of sodium trapped in the formation of the solid parts is approximately equal to the sodium in an equivalent volume of sea water.

(3) During the initial pumping out of a newly formed chamber almost $90 \%$ of the 'free' sodium and potassium which the chamber initially contained is removed with the water. If we compute the ratio:

$$
\frac{\text { Free sodium (mg) }}{\text { weight of water lost on drying (g) }}
$$

for the samples $(a)$ to $(e)$ we obtain the values: 10, $14,32,18$, Io respectively (sea water has a corresponding value of II). The sodium concentration is appreciably higher in the third chamber $(c)$ and in the anterior regions of the older chambers $(d)$, and this might suggest that water is extracted actively. However, the amounts of water in samples $(c)$ and $(d)$ were extremely small, I5 and $24 \mathrm{mg}$ respectively, and a small amount of evaporation during the collection of these samples may have taken place. These figures in no way invalidate the conclusion that the salt and water in a new chamber are largely removed together.

\section{The distribution of liquid in the cuttlebone}

Three methods were used to determine the distribution of liquid in a cuttlebone.

(I) A cuttlebone was cut sagitally into two halves and one half was placed cut side downwards in a tray containing a small depth of a solution of Sudan Black in xylene. This solution is sucked by capillary action into the dry regions of the bone but it does not penetrate into the wet regions. After about half an hour the bone was removed and examined. The black regions indicated those parts of the bone containing no water, the white regions those parts containing water.

(2) A longitudinal section about $\frac{1}{2} \mathrm{~cm}$ thick was cut from a cuttlebone and examined by transmitted light under a dissecting microscope. The regions containing water appeared lighter than those which were dry. The wet parts could be marked directly on the bone.

(3) The lamellae of the cuttlebone were scraped off one at a time and places where liquid was seen were marked on the outside wall of the succeeding chamber. ${ }^{2}$ We could distinguish (a) regions where no liquid could be seen, (b) regions in which liquid was visible, and (c) regions in which liquid freely welled up on scraping.

1 The figure of 10\% was found by an experiment on the chambered parts of the bone.

2 The newest chambers contain gas under relatively low pressure and it is possible that on puncturing a chamber air will rush in and perhaps displace liquid from its natural position. Usually, but not invariably, the newest chambers were punctured half way along their lateral margin. 
Each chamber is subdivided by about six thin chitinous membranes running parallel to the main walls of the chamber and examination of the distribution of liquid within a chamber, using method 2 above, showed that liquid extends from the siphuncular end by differing amounts in the different subchambers. By combining methods 2 and 3 on the same cuttlebone it was shown that condition $(c)$ corresponds to at least two of the seven or so subchambers being full of liquid.

Each method has some special advantage; the first is the quickest, the second allows the distribution of liquid within the subchambers to be seen, and the third ensures that the whole extent of each chamber is examined. All three methods gave the same general results. The first chamber, which is incomplete, is always full of liquid. The succeeding eight or so chambers are usually dry, whilst in the remaining chambers the liquid is all localized at the siphuncular ends of the chambers. The oldest chambers (i.e. those older than the thin chambers associated with the inflexion in the siphuncular surface) are usually very wet, and often almost completely filled with liquid. These chambers are only emptied when the bone becomes of exceptionally low density. In all chambers, apart from the first two, liquid, when present, increased progressively towards the siphuncular end. The siphuncular ends of the chambers were very wet indeed.

The most complete results are those given by the third method. This was applied to cuttlebones chosen to cover a large range of densities. The results for four of these cuttlebones are given in Text-fig. 2 where two quantities are plotted as ordinate; first the length of the chamber in the midline of the cuttlebone, and secondly that part of the chamber which contained liquid in sufficient quantity that it welled up on scraping (criterion $(c)$, above).

\section{The pressure of gas within individual chambers of the cuttlebone}

The pressures inside the individual chambers were studied in the following two experiments.

\section{Experiment $A$}

Freshly dissected cuttlebones were placed under sea water containing Sepia ink. The chambers were then opened under ink by slowly drawing a sharp edge along the centre of the siphuncular surface, i.e. along the line $x y$ of Text-fig. I. The bones were left for about an hour under ink and then taken out, dried gently and sawn up. Sections of such a bone are shown in Pl. I. It can be seen that ink has been drawn much more extensively into the newest chambers than into the others. The newest chambers are indeed almost completely filled with ink and must, before puncturing, have contained gas at very low pressures. Practically no ink has penetrated the older chambers $(z x)$; this is because these chambers are already almost full of water. 

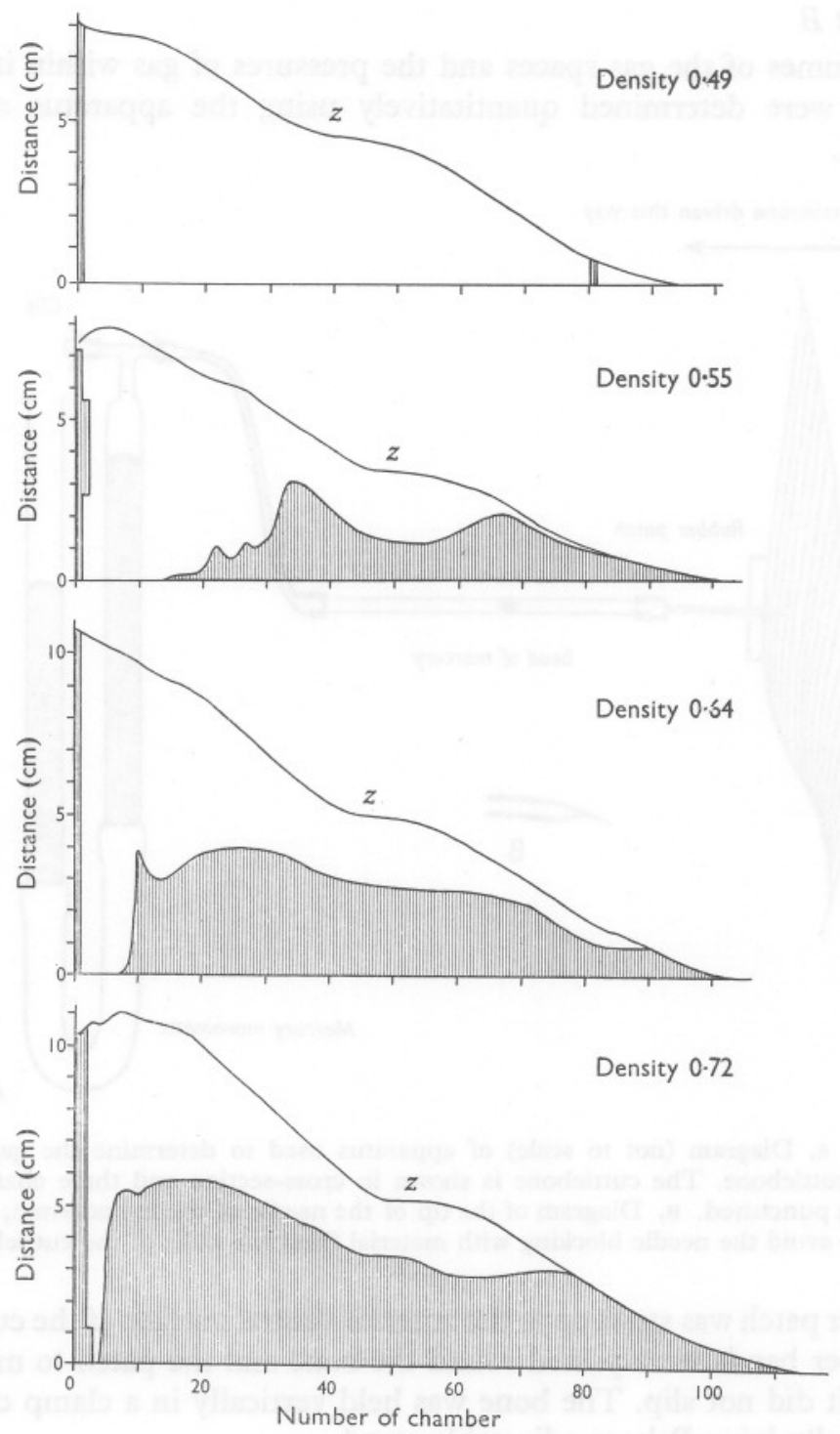

Text-fig. 2. Graphs showing the distribution of liquid in the chambers of four cuttlebones of widely differing densities. The ordinate represents distance along a chamber measured from the siphuncular surface, which therefore corresponds to the base line. The inflexion, $z$, marks the thin chambers of very small volume (see Text-fig. I). The regions containing liquid (category of (3)c, p. 369) are shown by the hatched areas. This category is such that at least two of the seven or so subchambers making up a chamber contain liquid. The number of subchambers filled with liquid increases progressively towards the siphuncular end. 


\section{Experiment $B$}

The volumes of the gas spaces and the pressures of gas within individual chambers were determined quantitatively using the apparatus shown in Text-fig. 3 .

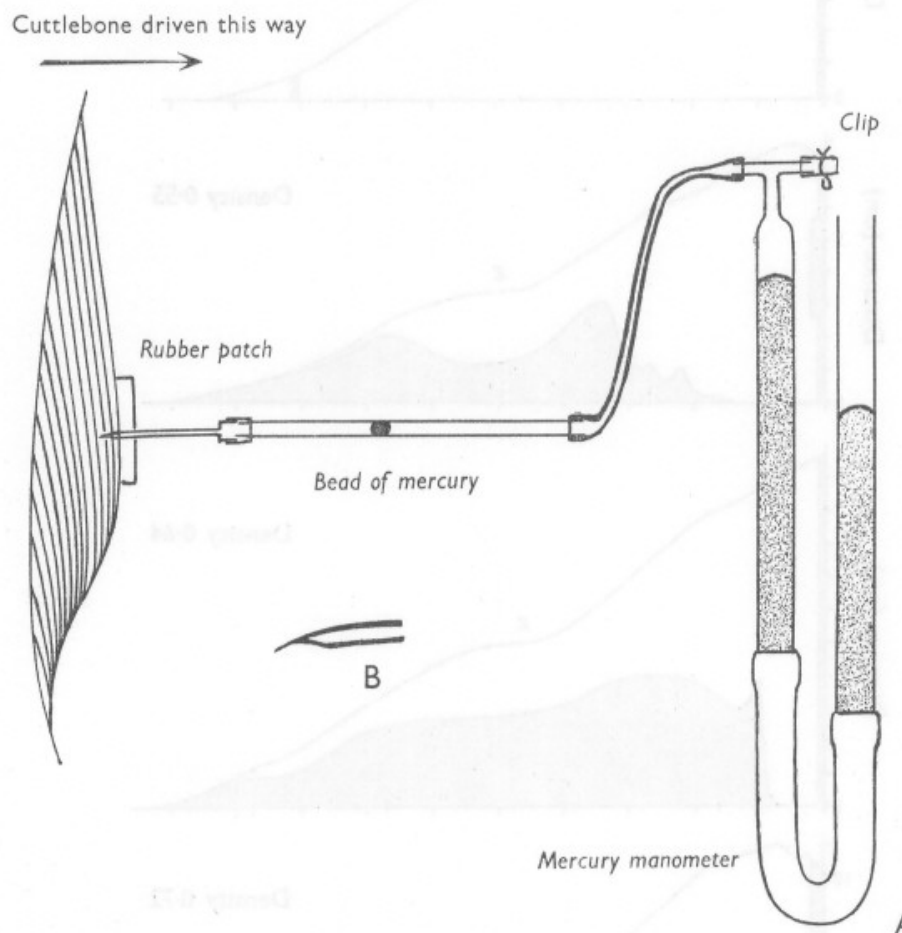

Text-fig. 3. A, Diagram (not to scale) of apparatus used to determine the gas pressures within the cuttlebone. The cuttlebone is shown in cross-section and three chambers have already been punctured. B, Diagram of the tip of the needle of the hypodermic; it has been bent over to avoid the needle blocking with material from the walls of the cuttlebone.

A rubber patch was stuck on to the anterior ventral surface of the cuttlebone. Two rubber bands were passed round the bone and the patch to make quite sure that it did not slip. The bone was held vertically in a clamp carried on a horizontally lying Palmer adjustable stand.

\section{Explanation of Plate I}

This cuttlebone has had its chambers punctured under sea water containing Sepia ink by a scratch along its siphuncular surface $(x y)$. A, A longitudinal section; B, a section showing the same cuttlebone after cutting away its anterior end to allow the lateral penetration of ink to be seen. Ink has been extensively drawn into the newer chambers which contain gas under low pressures. In this plate the cuttlebone is shown ventral side upwards. 

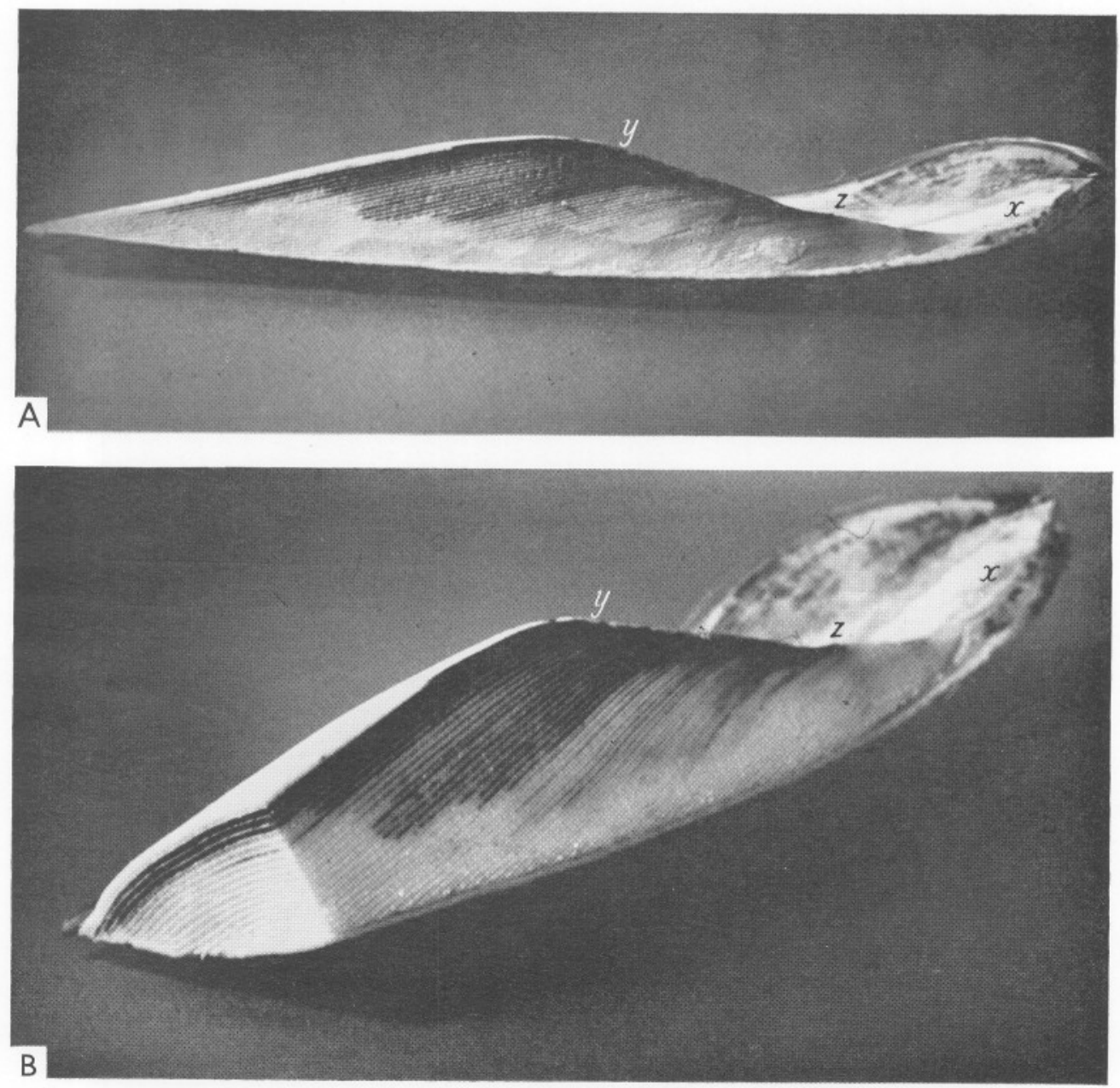

(Facing p. 372) 

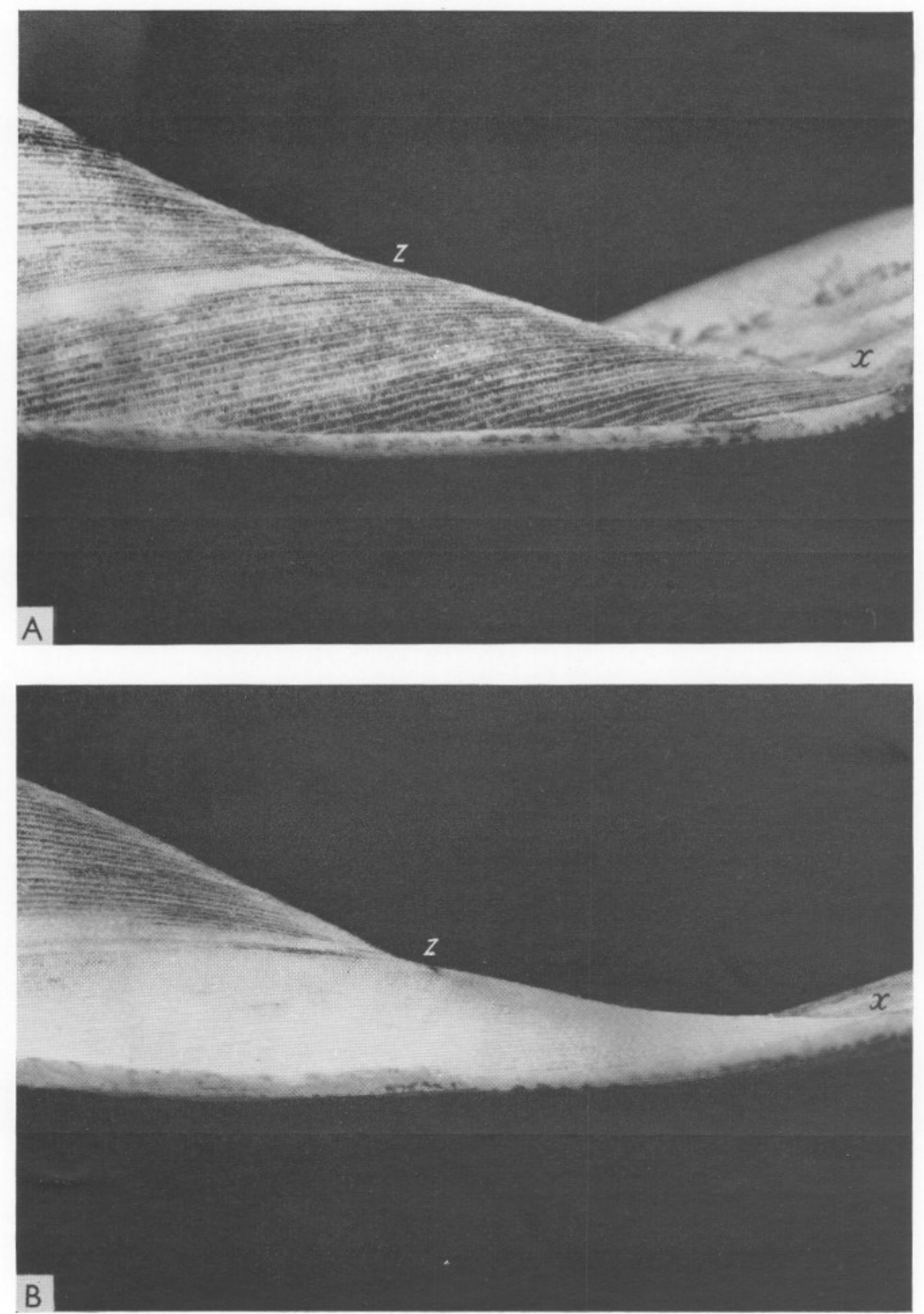
A I ml. graduated pipette was held horizontally in a second stand and this contained somewhere along its length a bead of mercury. The tapered end of the pipette was connected by a short piece of rubber tubing to a no. I4 hypodermic needle whose point had been bent as is shown in the detail B of Text-fig. 3. The other end of the pipette was connected by narrow bore tubing to a mercury manometer made of wide bore tubing. By raising and lowering one arm of the manometer the pressure of gas in the pipette could be varied in a known way.

The bead of mercury was brought close to the end of the pipette farthest from the open needle. The bone was now moved slowly on to the hypodermic needle so that this slowly punctured the bone through the rubber patch. (The tip of the needle had been bent so as not to be blocked by a neat core taken from the material of the cuttlebone.) The bead of mercury was watched carefully. Suddenly a marked displacement of this bead towards the needle was seen; the puncturing was then stopped. The needle had clearly penetrated into some gas space containing gas at less than atmospheric pressure.

Using the mercury manometer the gas pressure was varied and the positions of the mercury bead in the pipette found for various pressures.

These measurements finished, the cuttlebone was slowly driven farther on to the needle until a second sudden displacement of the mercury bead was observed. The puncturing was again stopped and volume and pressure measurements again made. This procedure was repeated a number of times, the needle being pushed farther and farther into the bone. Finally the needle was withdrawn. After finding the volume and density of the bone, this was sawn up and shaved gently with a scalpel to expose the path of the needle. The number of chambers punctured could then be counted. This number was equal to one more than the number of steps in pressure on puncturing. Since the newest chamber is incompletely formed and full of liquid this means that each change in displacement of the mercury bead corresponded to the puncturing of one chamber of the cuttlebone.

Text-fig. 4 shows a plot of volume change against the reciprocal of the pressure for the gas in the second chamber of a cuttlebone together with some gas in the measuring pipette. It can be seen that the points fall on a straight

\section{Explanation of Plate II}

A. A cuttlefish was kept in the dark for 2 days. Its cuttlebone which had then a low density (0.53) was punctured, under sea water containing Sepia ink, by a scratch along the siphuncular surface. The ink has penetrated very far into the older chambers (marked $z x$ ) showing that the pressure of gas within these chambers must have been very low before puncturing.

B. Similar to A except that the cuttlefish had not been kept in the dark. The cuttlebone's density was about 0.62 . Ink has not penetrated into the older chambers because these were already almost full of liquid. In this plate the cuttlebones are shown ventral side upwards. 
line, as Boyle's law would predict. This is true for the individual chambers of the cuttlebone. The volumes indicated by the application of Boyle's law correspond closely to estimates made of the volumes of the chambers from their physical dimensions.

It is now possible using Boyle's law to calculate the volume of the individual chambers and the pressures of gas within them before puncturing. The calculation which gives the volume of the gas space and the pressure of gas in the first chamber is given as an example.

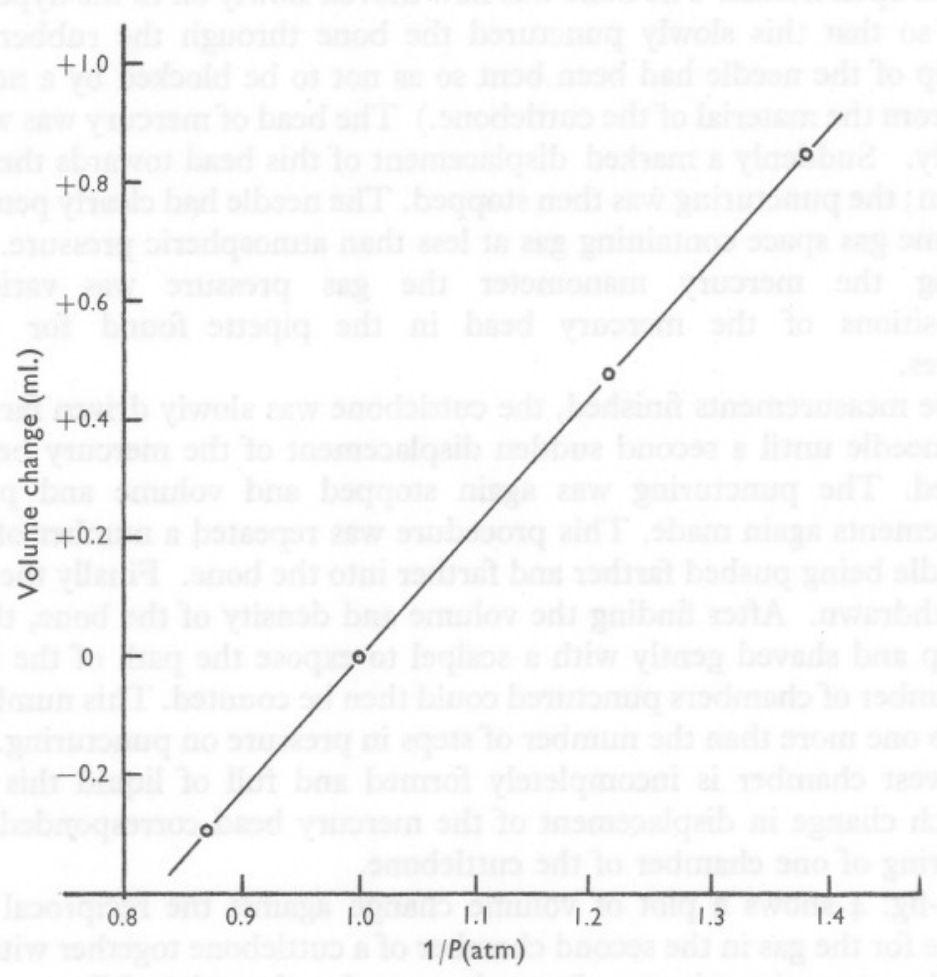

Text-fig. 4. Graph showing that the gas within the second chamber obeys Boyle's law. A straight line is given on plotting $\mathrm{I} /$ pressure against volume change.

Before puncturing let $V_{1}$ be the volume of the gas space within the first chamber, and $P_{1}$ the pressure of gas in this chamber, and let $V_{A}$ be the volume of gas between the tip of the needle and the mercury bead in the pipette, and $P_{A}$ the pressure of gas within the pipette.

After puncturing the first chamber let $V_{b}$ be the volume of the gas space between the tip of the needle and the mercury bead at pressure $P_{b}$ and let $V_{c}$ 
be a corresponding volume at the pressure $P_{c}$. Using Boyle's law, which was shown to be valid here, we may now write the equation:

$$
\left(V_{1}+V_{b}\right) P_{b}=\left(V_{1}+V_{c}\right) P_{c},
$$

and from this we can find $V_{1}$. We can now substitute the value for $V_{1}$ in the equation:

$$
P_{1} V_{1}+P_{a} V_{a}=\left(V_{1}+V_{b}\right) P_{b}=\left(V_{1}+V_{c}\right) P_{c},
$$

and so find $P_{1}$. This kind of calculation we can repeat for each successive puncturing and so find the volumes of gas spaces and the pressures within successive chambers. ${ }^{1}$
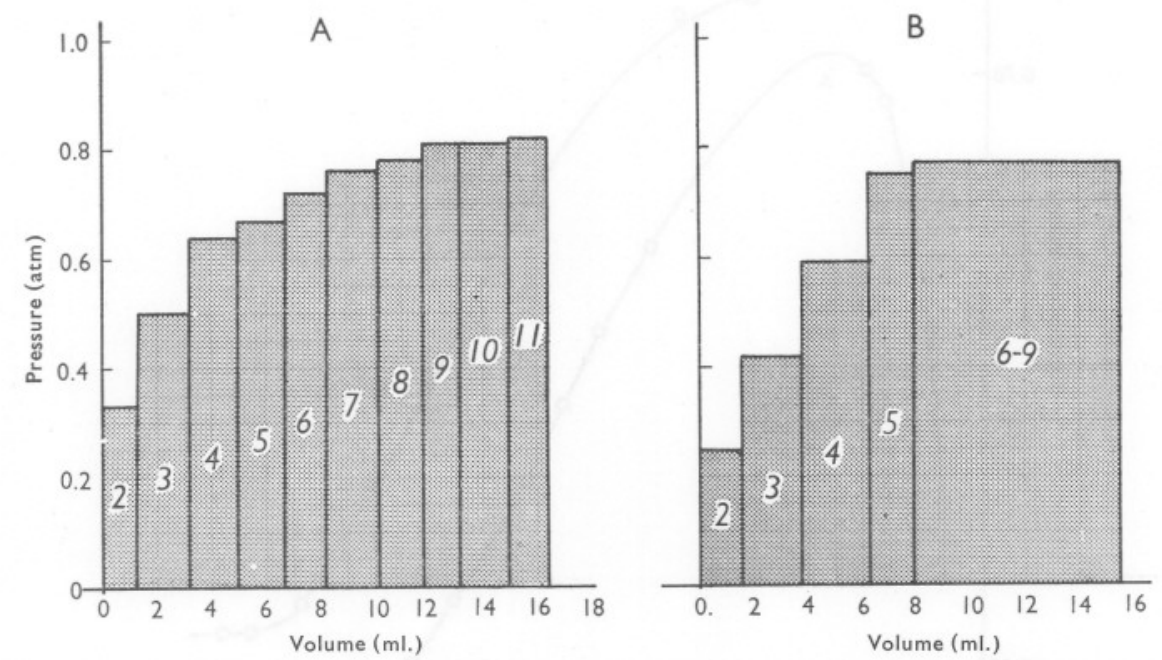

Text-fig. 5. Graphs showing as abscissa the cumulative volumes of the chambers of the cuttlebone starting from the most recently formed gas-filled chamber (chamber 2). The ordinate is pressure of gas within the individual chambers. A is for a cuttlebone in which the newest ro gas-filled chambers were punctured in turn. B is for a cuttlebone in which, after puncturing the first four gas-filled chambers individually, several were deliberately punctured together.

Text-fig. 5 shows results obtained in this way on two cuttlebones. Here the cumulative volumes of the chambers punctured are plotted against the pressures of gas within these chambers before puncturing. It can be seen that the pressure of gas is much lower in the newest chambers than in the somewhat older ones and that by the time we reach about the tenth chamber the pressures are close to the figure of about $0.8 \mathrm{~atm}$ found as the average pressure of gas within the cuttlebone (Denton \& Gilpin-Brown, I96r $a$ ).

1 The 'dead space' between the tip of the needle and the end of the pipette can be readily found. The needle is closed by pushing it into a rubber bung and the readings of the mercury bead along the pipette are measured for various pressures. The volume of 'dead space' and pipette is found by using Boyle's law. 


\section{The slowness of diffusion of gases into the cuttlebone}

To find at what rates gases within the cuttlebone will come to equilibrium with gases in the tissues around the cuttlebone a freshly dissected cuttlebone (Text-fig. 6, A) was placed in sea water saturated with carbon dioxide and through which carbon dioxide was being bubbled. Its changes of density with time were then found. A second cuttlebone (B) was soaked in sea water until

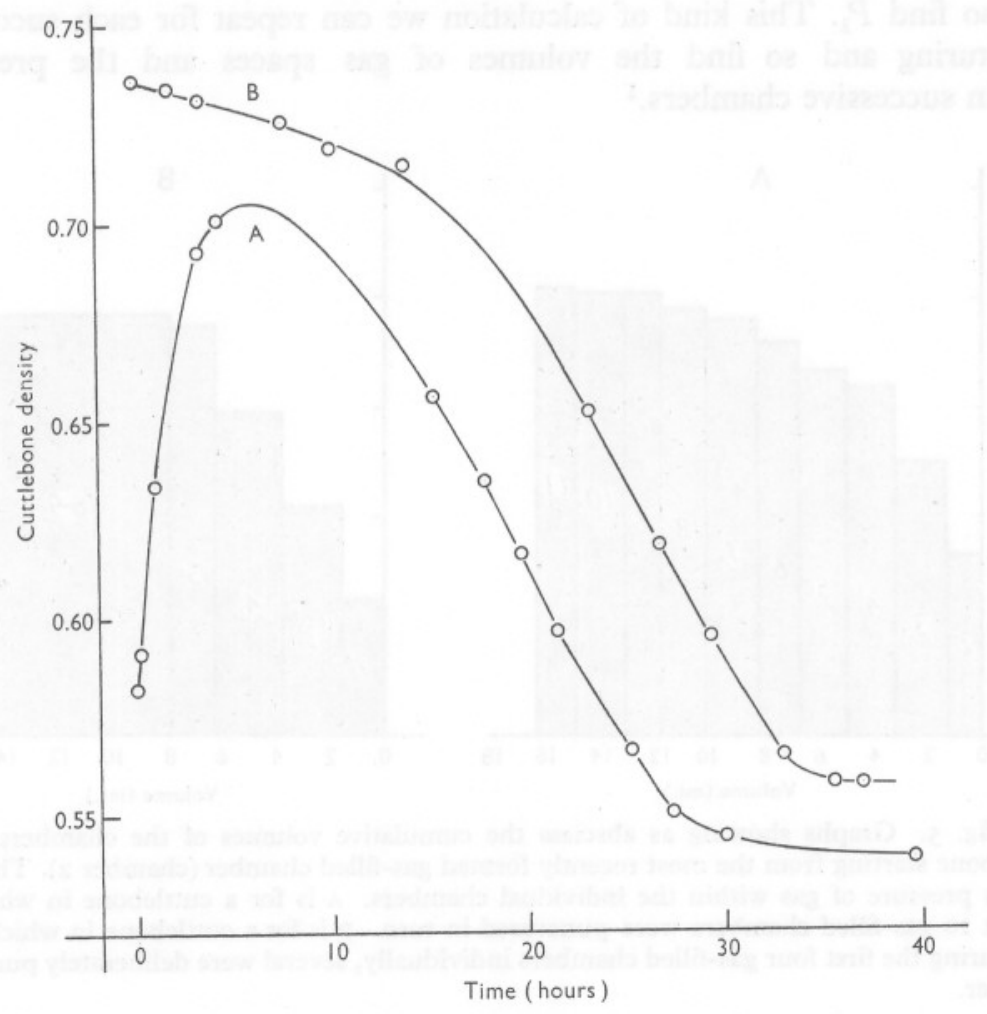

Text-fig. 6. An experiment to determine the rate of diffusion of $\mathrm{CO}_{2}$ into the cuttlebone. $\mathrm{A}, \mathrm{A}$ freshly dissected cuttlebone was placed in sea water saturated with $\mathrm{CO}_{2}$. $\mathrm{B}, \mathrm{A}$ cuttlebone was initially soaked in sea water until it reached constant density and then at time $\mathrm{ohrs}$ it was transferred to sea water saturated with $\mathrm{CO}_{2}$.

it attained constant density (Denton \& Gilpin-Brown, I96I $a$, fig. II) and then transferred to carbon dioxide saturated sea water. Carbon dioxide was chosen because it is much more soluble than either nitrogen or oxygen in sea water and will therefore come more quickly to equilibrium.

The results of this experiment are shown in Text-fig. 6. The curve A appears rather complicated but its form can be simply explained in terms of our knowledge of the cuttlebone. At first (as with the cuttlebones of figure II 
of Denton \& Gilpin-Brown, I96I $a$ ) water was pushed into the cuttlebone by the difference in pressure between the inside and outside of the bone. As this process slowed down the cuttlebone density then began to decrease, for carbon dioxide was all the time diffusing into the cuttlebone much more quickly than the gases inside could diffuse out. The gas pressure inside the bone therefore rose and water was expelled through the siphuncular surface. This process became increasingly fast as more and more liquid was expelled and the diffusion path for carbon dioxide became shorter; finally, when all the liquid available had been expelled from the cuttlebone its density remained almost constant, but the pressure of gas in the bone continued to rise. At the end of the period of soaking the cuttlebone was punctured and a stream of gas bubbles came from the hole, showing that the pressure of gas was above atmospheric. ${ }^{1}$ The curve B shows very similar results, but here the first phase, in which sea water is taken up, has been avoided by the initial soaking in sea water.

For convenience the numerical results of this experiment are given during the course of the discussion.

The slowness of gaseous diffusion into a cuttlebone was also shown by an experiment in which a cuttlefish was kept in the dark for 2 days so that its cuttlebone became of very low density, 0.53 . This cuttlebone was placed under Sepia ink and all its chambers were punctured in turn by a scratch along the siphuncular surface. It can be seen (Pl. II) that the ink has penetrated very deeply into the older chambers which are usually filled with liquid, but which in this cuttlebone will have just been pumped out. We thus see that the pressure of gas within these chambers was very low, and we have reproduced here the low pressures found in a newly formed chamber. In this case, of course, the liquid has certainly been pumped out from the siphuncular ends of the chambers, but we are not certain yet that this is the case for the newest chambers.

\section{Capillarity}

It seemed possible that surface tension forces (capillarity) might be used to ensure that liquid remained at the narrow siphuncular ends of the chambers, and we have already seen that a xylene solution is quickly drawn by capillarity into the narrow gas-filled space of the bone.

Cuttlebones, both fresh and dried, were sawn sagitally into two halves, and half cuttlebones were placed cut side downwards in a trough containing a shallow depth of a solution of aniline blue in water. Those parts of the chambers far away from the solution were punctured to make sure that the liquid could enter freely.

The watery solution of aniline blue was not taken up at all.

1 This experiment resembles that in which a porous pot filled with air is placed in an atmosphere of hydrogen at the same pressure. The hydrogen diffuses into the pot much more quickly than the air diffuses out, and the gas pressure inside the pot therefore rises. 
It therefore seems that the inside walls of the chambers of the cuttlebone are not very 'wettable' and we have no evidence that surface forces are used to localize liquid at the siphuncular ends of the chambers. This is in agreement with earlier conclusions (Denton \& Gilpin-Brown, 1961 $a$ ).

\section{DISCUSSION}

The slowness of equilibration of gases in the cuttlebone is partly because the distance for diffusion is great, but also because the large gas space into which the gases diffuse will hold, at a given pressure, a great deal more oxygen and nitrogen than will an equivalent volume of water. We can get some idea of how slow equilibration will be from the experiment on the uptake of carbon dioxide. Here, when the densities of the cuttlebones were about 0.6 , the rates of uptake of carbon dioxide were such that the times for the cuttlebones to move only half way towards equilibrium would have been about one day.

Now, for a given partial pressure difference, nitrogen diffuses in water at only about $\frac{1}{40}$ th the rate at which carbon dioxide diffuses ${ }^{1}$ (Hufner, cited by Krogh, I919) and so the half time of equilibrium for nitrogen would not be I but 40 days. This explains why, when the cuttlefish changes the volume of liquid within the cuttlebone, the mass of gas within the chambers remains almost constant for a long time. It also explains why the pressure of gas within the newest chambers is so low for these will have been formed recently and insufficient time will have elapsed for equilibrium to be attained. The distribution of pressures in the newest chambers (Text-fig. 4, p. 374) shows that it is not until we reach about the 9th chamber that equilibrium is approximately attained. Since the chambers are probably only laid down at a rate of three to five a month, this probably represents a period of 2 months, a very long time when we remember that these chambers are almost dry and the diffusion path for gases is relatively short.

Since differences in pressure of gas as well as differences in liquid content are maintained between neighbouring chambers, the principal walls of the chambers must be impermeable to both liquids and gases.

The pattern of water distribution in the cuttlebone has been shown to be a fairly simple one, and the following account summarizes the results both of the experiments which have been described here and many other observations on the cuttlebone. In all cuttlebones the newest chamber (no. I) is incomplete and filled with liquid. The second chamber, although often dry, may contain some liquid but this is rarely localized at the siphuncular end. In cuttlebones of density around $0 \cdot 6$ the oldest and most posterior chambers

\footnotetext{
1 The difference in diffusion rates arises principally because carbon dioxide is much more soluble than nitrogen in water so that for a given partial pressure difference there are many more molecules of carbon dioxide than nitrogen available for diffusion. Since the ratio of solubilities of nitrogen and carbon dioxide does not differ very much between pure water and sea water (Harvey, 1955) we may use the value of $\frac{1}{40}$ th here.
} 
are almost completely filled with liquid; whilst the second to about the Ioth chambers, that is those most newly formed, are dry. In very light cuttlebones (densities close to 0.5 ) there is very little liquid visible anywhere, and even the oldest chambers have been pumped dry. In very dense cuttlebones (densities close to 0.7 ) almost all the chambers, including most of those newly formed, contain liquid.

The distribution of liquid in cuttlebones of densities around 0.62 , i.e. densities close to the cuttlebone density which will make an animal neutrally buoyant is a rational one. The older chambers, which are very posterior, are kept fairly full of liquid, and the newer chambers, which lie more centrally along the length of the animal (Text-fig. 7), are kept dry. This means that

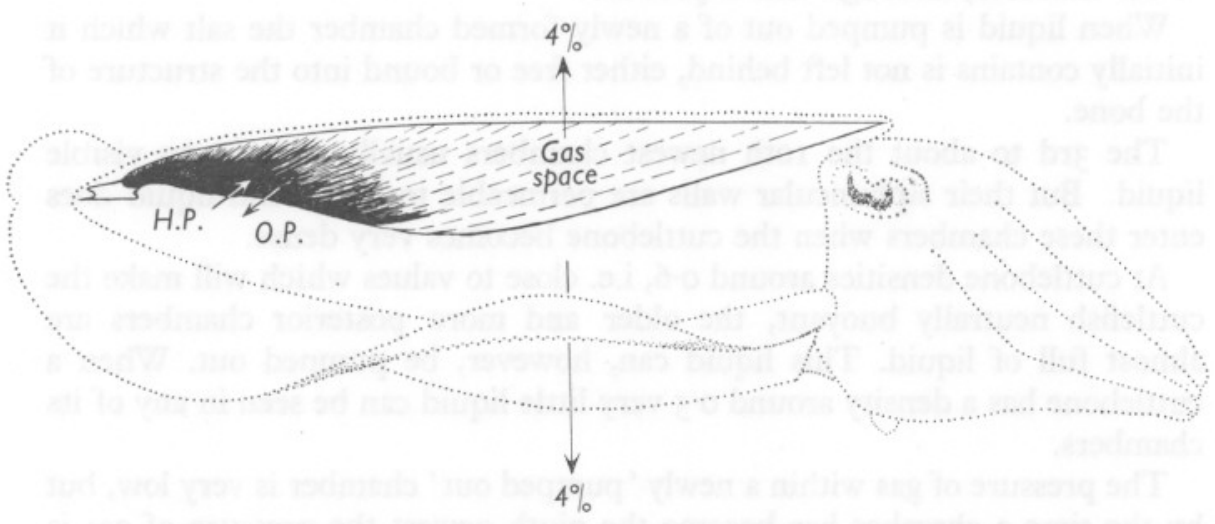

Text-fig. 7. Diagram summarizing our knowledge of the cuttlebone. The cuttlebone here represented has a density of about $0 \cdot 6$. Liquid within the cuttlebone is marked black. It can be seen that the oldest and most posterior chambers are almost full of liquid. If they were filled with gas, this would tend to tip the tail of the animal upwards. The newest Io or so complete chambers, which lie centrally along the length of the animal, are completely filled with gas. These chambers can give buoyancy without disturbing the normal posture of the animal.

The hydrostatic pressure (H.P.) of the sea is balanced by an osmotic pressure (o.P.) between cuttlebone liquid and the blood. In sea water the cuttlebone gives a net lift of $4 \%$ of the animal's weight in air and thus balances the excess weight of the rest of the animal. Note. The black markings in this figure have quite a different meaning from those of Plates I and II.

when the animal is approximately horizontal its centre of gravity is brought under its centre of buoyancy so that it can remain in this posture without effort. The cuttlefish can, however, both pump out the older chambers and let liquid into the newer ones. Since the main body of liquid lies at the siphuncular, i.e. posterior, ends of the chambers, when water is pumped out of the cuttlebone this will tend to bring the tail up. Having the tail slightly higher than the head is probably a good posture for the cuttlefish when it hunts over the sea bottom. It is certainly the posture which a cuttlefish takes in an aquarium tank as it prepares to attack a crab or prawn. 
We wish to thank Mr G. A. Battin for advice and help with the illustrations in this and the previous communications.

\section{SUMMARY}

The newest chamber of a cuttlebone is always incomplete and full of a watery solution. This contains sodium and potassium in concentrations close to those of sea water.

The second newest chamber contains a gas space and sometimes contains liquid. This liquid is rarely localized at the siphuncular end of the chamber. It is not certain that liquid is initially extracted through the siphuncular wall of the chamber, although this is possible.

When liquid is pumped out of a newly formed chamber the salt which it initially contains is not left behind, either free or bound into the structure of the bone.

The 3rd to about the Ioth newest chambers usually contain no visible liquid. But their siphuncular walls are permeable to liquid and liquid does enter these chambers when the cuttlebone becomes very dense.

At cuttlebone densities around $0 \cdot 6$, i.e. close to values which will make the cuttlefish neutrally buoyant, the older and more posterior chambers are almost full of liquid. This liquid can, however, be pumped out. When a cuttlebone has a density around 0.5 very little liquid can be seen in any of its chambers.

The pressure of gas within a newly 'pumped out' chamber is very low, but by the time a chamber has become the ninth newest the pressure of gas is close to the average value for the whole cuttlebone, i.e. about $0.8 \mathrm{~atm}$.

The low pressure of gas found in the newest chambers is explained by the slowness with which gas diffuses into a space created by the active removal of liquid.

It is estimated, from an experiment on the diffusion of carbon dioxide into the cuttlebone, that it would take over a month following a change in density of the bone, for the nitrogen in the bone to go half way to equilibrium with the nitrogen in the tissues. This explains why, when the cuttlefish changes the density of its cutlebone, the mass of gas within the cuttlebone remains almost constant.

The normal distribution of liquid within the cuttlebone is such that the cuttlefish can easily remain with its body horizontal in the sea. When the cuttlebone is made less dense, the change in the distribution of liquid will tend to tip the tail of the animal upwards. 


\section{REFERENCES}

Denton, E. J. \& Gilpin-Brown, J. B., I96I $a$. The buoyancy of the cuttlefish. F. mar. biol. Ass. U.K., Vol. 4I, pp. 319-342.

- 196I $b$. The effect of light on the buoyancy of the cuttlefish. F. mar. biol. Ass. U.K., Vol. 4I, pp. 343-350.

Denton, E. J., Gilpin-Brown, J. B. \& Howarth, J. V., I96I. The osmotic mechanism of the cuttlebone. F. mar. biol. Ass. U.K., Vol. 4I, pp. 351-364.

Harvey, H. W., 1955. The Chemistry and Fertility of Sea Waters. 224 pp. Cambridge University Press.

KROGH, A., I9I9. The rate of diffusion of gases through animal tissues, with some remarks on the coefficient of invasion. F. Physiol. Vol. 52, pp. 391-408. 